\title{
SEMBRAR DERECHOS. RECONFIGURACIÓN DEL TRABAJADOR RURAL COMO SUJETO DE DERECHO, EN LOS PROCESOS DE INTEGRACIÓN REGIONAL Y UNIVERSAL
}

TO SOW RIGHTS. RECONFIGURATION OF THE ARGENTINE RURAL WORKER, AS A SUBJECT OF REGIONAL AND UNIVERSAL RIGHT

\section{Daniela Verónica Sánchez Enrique*}

Resumen: Este artículo analiza la reforma del régimen de trabajo rural en Argentina, desde el debate teórico sobre la reconfiguración del trabajador, como sujeto de derecho universal, y la transformación del demos, frente a la normativa supraestatal generada por los procesos de integración regional y de globalización. El objetivo de este trabajo consiste en indagar sobre la incidencia de este proceso en el grado de incorporación de estándares internacionales de derechos humanos, en la normativa que regula el trabajo agrícola argentino. En el primer apartado se desarrolla el debate sobre las variaciones en el concepto de ciudadanía, a fin de repensar en qué medida incide en la reconfiguración jurídica del trabajador, de ciudadano nacional a sujeto de derecho regional y universal. En el segundo, se realiza un breve análisis de cierta normativa supranacional que manifiesta la internacionalización de los derechos de los trabajadores. Finalmente, se comentan los puntos estratégicos de la última reforma del régimen de trabajo rural argentino, procurando

\footnotetext{
*Abogada por la Universidad Nacional de La Rioja, Argentina. Doctoranda en Ciencias Sociales con mención en Humanidades de la Universidad Nacional de Quilmes. Becaria doctoral CONICET. Miembro del Centro de Estudios de la Argentina Rural de la Universidad Nacional de Quilmes, Buenos Aires, Argentina. danielasanchezenrique@gmail.com. Este trabajo fue realizado dentro del marco de una beca de CONICET y del programa ERASMUS, en la Facultad de Derecho de la Universidad de Salamanca, España, 2014-2015.
} 
destacar los principales cambios producidos por esta legislación en materia de incorporación de Derechos Económicos, Sociales y Culturales. La metodología seleccionada para el desarrollo de esta propuesta consiste en el análisis teórico y normativo, sobre la transformación del demos y de las normas referidas al nuevo régimen de trabajo agrario argentino (Ley Nacional 26.727), a los derechos humanos de los trabajadores en MERCOSUR, como organismo de integración regional, y del PIDESC.

Palabras clave: Derechos humanos, trabajo rural, integración regional, DESC, globalización

Abstract: This paper analyzes the reform of rural labor in Argentina, from the theoretical debate linked to the reconfiguration of the worker as a subject of universal rights and the transformation of demos, opposite the supranational rules generated by the processes of regional integration and globalization.The aim of this study is to analyze the impact of this process, rules and institutionally by the degree of incorporation of international human rights standards in the national legislation related to the new regime of Argentine agricultural work. The structure of this analysis contains three sections. In the first, the theoretical debate on the transformation of citizenship, caused by the tension between national legislation and international rights, to rethink the extent influences the transformation of the legal status of the worker, the subject develops subject to national law to regional and universal right. The second will make a brief analysis of some supranational law which manifests the internationalization of the rights of workers. Finally, we discuss the strategic points of the last reform of the Argentine rural work, trying to analyze the main changes. The selected for the development of this proposed methodology consists of theoretical analysis of the transformation of demos and regulations for the new regime of Argentine farm work (National Law 26,727), which will be supplemented by referring to bodies of Latin American regional integration and International Treaties on Human Rights, specifically on Economic, Social and Cultural Rights (ESCR).

Key words: Humans Rights, Rural Labor, Regional Integration, ESCR, Globalization 
Sumario. I. Introducción. II. Reconfiguración del trabajador como sujeto de derecho regional y universal desde la perspectiva de los derechos humanos. III. Internacionalización de los derechos de los trabajadores. III.1. Trabajadores como sujetos de derecho universal. PIDESC. III.2. Trabajadores como sujetos de derecho regional. MERCOSUR. IV. Nivel de incorporación de DESC en la reforma del régimen de trabajo agrario Argentina, Ley 26.727. IV.1. Régimen especial y/o aplicación de la LCT para el trabajo agrícola. IV.2. Trabajadores incluidos y excluidos. IV.3. Contrato de trabajo agrario. IV.4. Modalidades de contratación. IV.4.1. Contrato de trabajo permanente continuo. IV.4.2. Contrato de trabajo temporario. IV.4.3. Contrato permanente discontinuo. IV.4.4. Prohibición de trabajo infantil. V. Derechos vinculados a la seguridad social. V.1. Preaviso. V.2. Indemnización por antigüedad. V.3. Remuneraciones. V.4. Beneficio jubilatorio. VI. Registro Nacional de Trabajadores y Empleadores Agrarios (Renatea). VII. Conclusiones. Referencias.

\section{INTRODUCCIÓN}

Los estudios sobre trabajo agrícola en Argentina, durante las últimas décadas, analizaron las transformaciones ocasionadas por el proceso de globalización e integración regional, vinculadas a la estructura productiva, la población y la tecnología. Los principales aportes en este sentido se realizaron sobre el fenómeno de transnacionalización de la agricultura, desde perspectivas económicas (Neiman \& Quaranta, 2000) y sociológicas (Neiman, 2001; Bendini, Radonich \& Steinberguer, 1998 y Rau, 2012).

Este interés académico responde a que:

«Desde finales de los años 80 en Argentina, al igual que en otros países de América Latina, sucedieron reformas estructurales destinadas a establecer nuevos ordenes caracterizados por la desregularización y liberalización de los mercados y apertura de la economía, lo que produjo un aumento en el comercio internacional y de las inversiones extranjeras directas en distintas ramas de la actividad económica, entre las que se encuentran el caso de las agroindustrias. Este proceso de transformación global modificó no solo el entorno macroeconómico, sino también las 
estructuras productivas y las relaciones de las distintas agendas económicas tanto entre si, como con otros países y con el conjunto de la economía mundial» (Garrido \& Peres, 1998, pág.1).

No obstante, los aspectos de esta transformación han sido escasamente analizados desde la perspectiva de los Derechos Económicos Sociales y Culturales (en adelante DESC). Por esa razón, este artículo pretende realizar un aporte desde la dimensión los derechos humanos, que entiende a los trabajadores como el centro de su estudio, no como variables de producción, sino como actores estratégicos del mundo agrícola.

El objetivo de este trabajo consiste en analizar el grado de incorporación de estándares internacionales de derechos humanos, en la última reforma del régimen de trabajo agrícola argentino, Ley 27.626 (2013). Para lo cual, se retomará el debate teórico sobre la reconfiguración del trabajador como sujeto de derecho regional y universal, producto del cambio en el concepto de ciudadanía (demos), generado por la normativa supranacional de los procesos de globalización e integración latinoamericana.

La relevancia de esta problemática se justifica porque existen centros de decisión política situados más allá del poder estatal (FMI, Grupo de París, capitales transnacionales), que inciden tanto en los poderes públicos y privados, como sobre la sociedad civil en general y los trabajadores en particular. En consecuencia, el Estado nacional ya no es el único centro de adopción de decisiones vinculantes que permiten la regulación de las relaciones laborales. Estos centros de poder pueden actuar sin los límites que las Constituciones modernas impusieron al poder dentro de las fronteras de cada Estado, lo que cuestiona las ideas tradicionales sobre el demos y el cratos del constitucionalismo clásico.

En este punto, conviene considerar en qué medida este nuevo escenario reconfigura la situación jurídica del trabajador como sujeto de derecho, desde la escala nacional a la supraestatal. Esto sucedería en dos dimensiones, tanto por los procesos de integración regional como por la asimilación de los tratados internacionales de 
derechos humanos a la Constitución Nacional, en el caso argentino en 1994 (art. 75, inc. 22).

De este contexto, se infiere que para garantizar los derechos de los trabajadores, su problemática debería abordarse desde la perspectiva transversal de los derechos humanos. Frente al fenómeno de la transnacionalización de la agricultura y de los capitales multinacionales, el análisis jurídico de esta materia también debería realizarse desde un enfoque de derecho supranacional, específicamente de DESC.

La estructura de este artículo, estará dividida en tres apartados. En el primero, desarrollaremos el debate teórico sobre la transformación del concepto de ciudadanía, ocasionado por la tensión entre la normativa nacional y la internacional. Con el objetivo de indagar en qué medida reconfigura la situación jurídica del trabajador, de sujeto de derecho del plano nacional al nivel regional y universal. En el segundo, analizaremos como esa reconfiguración teórica se manifiesta en un proceso de incorporación de estándares internacionales de derechos humanos vinculados a la condición de trabajador, a nivel regional. Con este fin, realizaremos un breve análisis de cierta normativa supranacional (del PIDESC y de normas específicas del MERCOSUR) que manifiesta la internacionalización de los derechos de los trabajadores.

Finalmente, con el objeto de analizar ese mismo proceso a nivel nacional, comentaremos los puntos estratégicos de la última reforma del régimen de trabajo rural argentino, procurando identificar los principales cambios producidos en materia de incorporación de estándares internacionales de derechos humanos, en general, y de DESC, en particular.

\section{RECONFIGURACIÓN DEL TRABAJADOR COMO SUJETO DE DERECHO REGIONAL Y UNIVERSAL DESDE LA PERSPECTIVA DE LOS DERECHOS HUMANOS}


Limitar el estudio de la incorporación de estándares internacionales de derechos humanos a la normativa específica sobre trabajo rural en Argentina, sería insuficiente para cumplir con el objetivo de este artículo. Por ello, se analizará a continuación el debate teórico sobre la transformación del concepto de ciudadanía (demos), ocasionado por la tensión entre la normativa nacional y la internacional, a fin de repensar en qué medida incide en la situación jurídica del trabajador, en su reconfiguración como sujeto de derecho del plano nacional, al nivel regional y universal.

De este modo, el fenómeno de la globalización y los incipientes procesos de integración regional, generan un nivel de supraestatalidad que influye en la reconfiguración de los sujetos de derecho, en este caso, de los trabajadores. Este fenómeno cuestiona la exclusividad de la normativa del Estado Nación.

Las relaciones económicas y sociales que emergen de la voluntad política de los Estados de constituir, por ejemplo, un Mercado Común del Sur (MERCOSUR), la Unión de Naciones Suramericanas (UNASUR) y la Comunidad de Estados Latinoamericanos y Caribeños (CELAC), presuponen la expansión de las economías nacionales a nivel regional y en consecuencia, el avance en la libre circulación de trabajadores, capitales, bienes y servicios que conforman la integración regional plena.

En este sentido, la globalización no se reduce a un fenómeno de carácter económico, es también político, tecnológico y cultural. Se constituye como una serie compleja de procesos que no solo se manifiesta cuestionando la posición del Estado hacia arriba, más allá de sus fronteras, superándolo, y hacia abajo, dentro de sus límites geográficos, cuestionando su capacidad de cohesión interna.

En el primer caso, esto se traduce jurídicamente como una crisis de las constituciones como normas supremas del Estado nación, y en el segundo, en la necesidad de incorporar los estándares internacionales de derechos humanos a las legislaciones nacionales. Este proceso, podría ser denominado como internacionalización de la legislación interna. 
Los fenómenos de la revolución tecnológica e informática, de globalización y los procesos de integración regional, generan la necesidad de adaptar los conceptos del constitucionalismo moderno a esta serie de transformaciones estructurales que se traducen en una crisis constitucional.

Esta es:

«una situación en la que se combinan tres rasgos básicos: un ambiente cultural en completa evolución; un replanteamiento y transformación de las funciones del Estado y en el ámbito propiamente jurídico una reacción contra el método positivista anterior originando un enorme pluralismo en cuanto a los métodos, los objetivos y los presupuestos conceptuales del constitucionalismo y del derecho constitucional que introducen una confusión en la que resulta difícil encontrar sólidos puntos de apoyo» (Bustos Gisbert, 2005, pág. 76).

En este punto, conviene retomar el debate teórico sobre la reconfiguración de los dos elementos esenciales en cualquier Estado de derecho. En primer lugar, la transformación de la normativa constitucional como ley suprema, en tanto que límite a los poderes políticos (cratos). En segundo término, la tensión en la idea de pueblo o ciudadanía (demos), y de la transformación jurídica de la dimensión de trabajador, como sujeto de derecho regional y universal.

De esta manera,

«los procesos de cambio ponen a prueba las funciones de certeza y seguridad que son exigibles de cualquier sector del ordenamiento jurídico (...) Lo cual termina repercutiendo en los procesos de interpretación y aplicación judicial del derecho constitucional» (Cascajo, 2003, pág. 81).

En este sentido, cierta doctrina (Haberle, 2002; Ackerman, 1998 o Ferrajoli, 2003), ha repensado los presupuestos constitucionales a la luz de las transformaciones del nuevo milenio, signado por diversos procesos de integración regional. Con respecto al debate sobre la reconfiguración del cratos, Walker, en 
su construcción sobre la idea de pluralismo constitucional, señala ciertos puntos críticos de reconfiguración constitucional de los que se mencionarán tres a continuación (Walker, 2002).

La primera se refiere al estatismo de la idea de constitución. Fundamentalmente, critica el discurso que defiende la identificación ontológica entre Estado y Constitución Nacional. El constitucionalismo moderno entendía que el marco estatal era capaz de contener la dinámica y la estructura del poder político, económico y social. No obstante, en la medida en que esa dinámica comienza a exceder al Estado y sus fronteras, la Constitución y sus presupuestos deben ser repensados a la luz del fenómeno de la transnacionalización.

La segunda se relaciona con lo que Walker denomina "fetichismo constitucional", que implica el discurso que sobredimensiona la capacidad de la Constitución para regular y canalizar la política, la economía y la sociedad, ya que esa certeza es un obstáculo para visibilizar los nuevos desafíos que deben ser resueltos. En este sentido, Ward habla del nuevo opio de los pueblos y denuncia la incapacidad del discurso constitucional de contener al proceso político como subsistema dentro del sistema jurídico-constitucional (Ward, 2001, pág. 28).

Al mencionar el sesgo normativo de la Constitución, cuestionando su supuesta neutralidad ideológica, Walker alega que su construcción responde a un modelo con una ideología predominante, sin negar sus matices, que favorece a determinados intereses y valores, y que impide su comprensión como un campo de juego leal para que una pluralidad de aspiraciones sociales e ideológicas pueda contenerse.

Finalmente, se hará referencia a la problemática sobre la vigencia de los conceptos constitucionales, a la que necesariamente conducen las anteriores, y que concluye en que la eficacia del derecho constitucional depende del replanteo de ciertos presupuestos. 
En este contexto, los problemas y las críticas que más interesantes son las relativas al cambio de paradigma del Estado nación, a la aparición de centros de poder político más allá de él, y a los efectos que estos procesos producen en categorías constitucionales como la de "demos", que se traduce individualmente como ciudadano y trabajador.

Sobre este punto, Habermas sostiene que una comprensión teórica-comunicativa de la democracia no puede descansar sobre una idea concéntrica de pueblo, entendido como identidad étnica, porque se presentaría como homogéneo algo que, en sí mismo, es muy heterogéneo (Habermas, 1997, pág. 262).

De este modo, este debate teórico se ve reflejado en el incipiente proceso de unidad en Sudamérica que avanza integrando económica, jurídica y administrativamente a los Estados, pero también a los ciudadanos latinoamericanos en organismos regionales como MERCOSUR, UNASUR y CELAC. Esto se ha desarrollado dentro de un marco de contexto cultural, y de una experiencia histórica común, de manera que podría hablarse de una unidad regional que incluye las diversidades nacionales.

Sobre este debate, existe un aporte académico interesante de Weiler (1997), desarrollado en el contexto de integración regional de la Unión Europea. Este argumento nace como reacción a la sentencia del Tribunal Constitucional alemán que rechaza la aplicación de una norma de derecho comunitario, alegando que su soberanía nacía de su identidad como pueblo alemán.

En este sentido, Weiler plantea que la unión típica del constitucionalismo moderno entre volk (Nación), demos (Pueblo) y Estado, no solo es innecesaria, sino que es inaplicable en los nuevos Estados de derecho contemporáneos, en un contexto de procesos de integración regional. Con esta postura no pretende anular la categoría de nacionalidad sino cuestionar que sea la exclusiva condición para la plena pertenencia cívica y política a una organización política determinada, pensada en términos de supraestatalidad. 
De la separación de los conceptos de nacionalidad y ciudadanía, puede hablarse de la coexistencia de múltiples demoi (pueblos). Desde esta nueva perspectiva cada ciudadano puede pertenecer institucional y jurídicamente a un Estado determinado, a una región y a una entidad supranacional, al mismo tiempo. En este sentido podría afirmarse que:

«esta perspectiva permite al hombre considerarse a sí mismo, como perteneciente simultáneamente a varios demoi, basados en diferentes factores subjetivos de identificación: nacional en el sentido de orgánico cultural, y a la vez ciudadano europeo en términos de afinidades transnacionales respecto a valores compartidos que trascienden la diversidad etno-nacional» (Bustos Gisbert, 2005, pág. 127).

Este argumento, implicaría que un ciudadano en ciertos ámbitos de su vida pública esté dispuesto a aceptar la legitimidad y la autoridad de decisiones adoptadas por entidades supranacionales latinoamericanas, en su rol como ciudadano latinoamericano, aún sin haberlas elegido por su voto y sin dejar de ser, ni sentirse, ciudadano nacional. Lo que no implica negar la importancia de los Estados sino asumirlos e integrarlos como elementos, esenciales y soberanos, de organizaciones supranacionales.

La transformación del concepto de ciudadanía nacional que convive con el de ciudadanía regional y universal, tiene una incidencia directa en la condición de los trabajadores, ya que han dejado de ser sujetos de derecho solo dentro del marco del Estado nación,(régimen de trabajo agrícola) para convivir con su dimensión de sujetos de derecho regional (régimen de MERCOSUR), y universal (DESC).

Luego de haber desarrollado el debate teórico sobre la transformación del concepto de ciudadanía, en el siguiente capítulo, se analizará como esa reconfiguración teórica se presenta jurídicamente, de una manera concreta. Es decir, como se manifiesta en un proceso de incorporación normativa de derechos humanos al mundo del trabajo, tanto a nivel internacional como 
regional, y que al mismo tiempo reconocen a estos derechos como vigentes y exigibles en los Estados nacionales.

Con este fin, se realizará un breve análisis de cierta normativa supranacional, tanto del MERCOSUR como del PIDESC, que manifiestan la internacionalización de los derechos de los trabajadores y confirman su incipiente transformación como sujetos de derecho, regional y universal, en materia de derechos humanos.

\section{INTERNACIONALIZACIÓN DE LOS DERECHOS DE LOS TRABAJADORES}

\section{III.1. Trabajadores como sujetos de derecho universal. PIDESC}

La importancia del enfoque transversal de derechos humanos radica en visibilizar, más allá de los derechos laborales, las condiciones de vida y la vulnerabilidad social de los trabajadores, ya que éstas, son las que definen su capacidad de negociación laboral, en un mercado configurado por la confrontación de intereses entre capital y trabajo. El proceso por el que los DESC adquirieron el status de derechos humanos es inverso al de los civiles y políticos, ya que su consagración internacional, como tales, fue posterior a la constitucional de los Estados (González Morales, 2013).

De este modo, se generó la necesidad de incorporar los estándares internacionales de DESC a la legislación nacional, en general, y al régimen de trabajo agrícola, en particular. En este sentido, la reforma constitucional argentina de 1994 otorga a los tratados internacionales de derechos humanos, jerarquía constitucional (art. 75, inc. 22). Esta modificación fortaleció la integración de Argentina dentro del Sistema Interamericano de Derechos Humanos que entiende que los DESC tienen naturaleza obligatoria y no solamente programática.

En las últimas décadas, con el fortalecimiento de las democracias latinoamericanas, la Corte Interamericana de 
Derechos Humanos, no solo resuelve como tribunal de apelación supraestatal, casos vinculados a derechos humanos de naturaleza civil (por ejemplo: el voto, la libertad de expresión, la privación ilegitima de la libertad, entre otros), sino que se han incrementado casos sobre DESC, relacionados no solo con la consolidación sino también con la calidad de la democracia en un sentido más amplio, mediante la garantía de los derechos sociales.

En este sentido, la Comisión Interamericana de Derechos Humanos, definió los "Lineamientos para la elaboración de indicadores de progreso en materia de DESC", publicados en el año 2008. Esta resolución sienta las bases para diseñar la medición de dicho progreso, a fin de posibilitar la generación de políticas públicas eficaces para cada sector.

Los derechos humanos de los trabajadores se encuentran explícitos en el PIDESC adoptado por la Asamblea General de la ONU en 1966. Dicho pacto consagra el derecho a un trabajo libremente escogido, estableciendo para los Estados partes la obligación de adoptar medidas adecuadas para garantizar este derecho, orientadas a lograr un desarrollo económico, social y cultural constante y la ocupación plena y productiva, en condiciones que garanticen las libertades políticas y económicas fundamentales de las personas (PIDESC,art. 6). También se reconoce el derecho de toda persona al goce de condiciones laborales que le aseguren en especial: a) Una remuneración que proporcione como mínimo a todos los trabajadores: i) Un salario equitativo; ii) Condiciones de existencia dignas para ellos y para sus familias (PIDESC, art. 7). Con respecto a los derechos sindicales, son reconocidos por el PIDESC en el art. 8.

Sobre el efectivo cumplimiento y exigibilidad del PIDESC, en su art. 2, inc. 1, incorpora lo que se ha denominado como cláusula de progresividad. Esta disposición determina que los Estados que adhieran al pacto, entre los que se cuenta Argentina, se comprometen a adoptar medidas, especialmente económicas y 
técnicas, hasta el máximo de los recursos de que dispongan, para lograr progresivamente, el respeto de los $\mathrm{DESC}^{1}$.

\section{III.2. Trabajadores como sujetos de derecho regional. MERCOSUR}

Las migraciones internacionales de trabajadores agrícolas son un fenómeno histórico pero, frente al proceso de globalización actual, requieren ser pensados desde categorías que trasciendan las fronteras del Estado nación, ya sea dentro del marco de los procesos de integración regional latinoamericana (MERCOSUR, UNASUR, CELAC) como de organizaciones supra-regionales como la Organización de Naciones Unidas (ONU) y la Organización de los Estados Americanos (OEA). En nuestro caso, nos concentraremos en el MERCOSUR por tener mayor desarrollo institucional y por encarnar los principales desafíos en materia de integración regional en la materia que nos ocupa.

En este contexto, se ha reforzado la obligación a los Estados partes del MERCOSUR de asegurar un trato no discriminatorio y que resguarde los derechos humanos de las personas en general, y de los trabajadores migrantes en particular. En este sentido, el derecho a migrar es considerado un derecho humano. Desde 1948, el art. 13 la Declaración Universal de Derechos Humanos establece que «toda persona tiene derecho a circular libremente y a elegir su residencia en el territorio de cada Estado. Toda persona tiene derecho a salir de cualquier país, incluso del propio, y a regresar a su país». En Argentina, la Ley de Migraciones $\mathrm{n}^{\circ}$ 25.871, sancionada en 2003 (reglamentada en 2010) lo consagra expresamente en su art. 4 al afirmar que «el derecho a la migración

\footnotetext{
${ }^{1}$ Protocolo de San Salvador (2005), art. 5.1: «A los fines de este documento por principio de progresividad se entenderá el criterio de avance paulatino en el establecimiento de las condiciones necesarias para garantizar el ejercicio de un derecho económico, social o cultural. Con posterioridad, define como "medidas regresivas a todas aquellas disposiciones o políticas cuya aplicación signifique un retroceso en el nivel de un goce o ejercicio de un derecho protegido».
} 
es esencial e inalienable de la persona y la República Argentina lo garantiza sobre la base de los principios de igualdad y universalidad». A esta normativa se suma la Ley General de Reconocimiento y Protección al Refugiado ( $\mathrm{n}^{\circ} 26.165$ ) de 2006 y el programa Patria Grande (2005), destinado a la regularización de la situación de inmigrantes en Argentina.

Es indiscutible que este ius migrandi implica no solo el derecho de los individuos de elegir su lugar de residencia en cualquier Estado, sino también la obligación emergente de éste de acogerlo como nuevo ciudadano, si la residencia es permanente, o bien, de respetar sus derechos en el caso de que sea temporaria. La ley contempla la posibilidad de irregularidad de los individuos migrantes dentro de un país diferente del de nacimiento que, en general, se da en el marco de su condición de trabajadores.

De este modo lo ha entendido la Corte Interamericana de Derechos Humanos al pronunciarse sobre el caso Hoffman Plastic Compounds v. National Labor Relations Board, en la Opinión Consultiva No 18 (17-IX-2003). En ella dispuso que la situación de irregularidad migratoria de una persona, no podía ser causa de la privación de sus derechos laborales.

Dentro del marco orgánico del Mercosur los espacios que tratan la materia de las migraciones y la movilidad laboral desde sus diferentes perspectivas y con diversos niveles de desarrollo son: a) el Subgrupo $\mathrm{n}^{\mathrm{o}} 10$ de Asuntos Laborales, Empleo y Seguridad Social; b) la Comisión Sociolaboral del Mercosur; c) el Grupo de Liberalización del Comercio de Servicios; d) el Foro Consultivo Económico y Social; e) la Reunión de Ministros del Interior; y f) el recientemente creado Grupo "ad hoc" sobre Integración Fronteriza.

Entre ellos se destaca el Subgrupo $\mathrm{n}^{\circ} 10$ de Asuntos Laborales, Empleo y Seguridad Social. Este es un órgano que reporta al Grupo Mercado Común y funciona tripartitamente. Sus trabajos se desarrollan sobre la base de la consulta entre los sectores gubernamentales, sindicales y empresariales de cada país y proponen medidas tendientes a coordinar políticas nacionales en 
todos los países de MERCOSUR. En segundo lugar, se ubica la Comisión "ad hoc" que tiene como objetivo el estudio de las condiciones en que se desarrolla el trabajo en las regiones fronterizas nacionales, esencial para la formulación políticonormativa de casos especiales.

Como manifestación legislativa sancionada dentro en este contexto, destacamos el Acuerdo Multilateral de Seguridad Social (1997), que reconoce:

«a los trabajadores que presten o hayan prestado servicios en cualquiera de los Estados Partes reconociéndoles, así como a sus familiares y asimilados los mismos derechos y estando sujetos a las mismas obligaciones que los nacionales de dichos Estados Partes con respecto a los específicamente mencionados en el presente Acuerdo».

Finalmente, el Proyecto de Acuerdo sobre Residencia para Nacionales de los Estados Partes del Mercosur, aprobado en 2002, declara la igualdad de derechos civiles, sociales, culturales y económicos entre nacionales del país de recepción y nacionales de los otros países firmantes (art. 9, 1) y refuerza el principio de reunión familiar (art. 9,2); el derecho a transferir remesas a su país, y el derecho de los hijos de los inmigrantes a tener un nombre, una nacionalidad y a acceder a la educación, aún en caso de que sus padres estén en situación irregular. De este modo, el acuerdo actualmente en gestión responde ajustadamente a principios sustentados tanto desde los Convenios 97 y 143 de la OIT, como desde la Convención de 1990 de las Naciones Unidas.

La incorporación de ciertos estándares internacionales de derechos humanos vinculados a los trabajadores, en este caso de DESC, por uno de los más desarrollados organismos de integración regional (MERCOSUR), del cual Argentina forma parte, constituye el primer ejemplo de reconfiguración del trabajador rural como sujeto de derecho regional. Este marco de protección supranacional de los derechos humanos de los trabajadores se hace efectivo por haber sido reconocido como prioritario en la agenda de latinoamericana de integración regional. 
Como manifestación institucional de este objetivo podemos mencionar al Instituto de Políticas Públicas y Derechos Humanos del MERCOSUR, en el que se hace mención al derecho a no migrar. Es decir que, dentro de un marco de cooperación regional, comienza a pensarse la problemática del trabajo migratorio, también desde la obligación de los Estados parte de generar condiciones de respeto de DESC para que sus habitantes no se vean forzados a migrar por razones de pobreza estructural.

En todo lo expuesto hasta este punto, se analizó el grado de reconocimiento de los derechos humanos de los trabajadores en dos marcos normativos estratégicos, tanto a nivel regional (MERCOSUR), como universal (PIDESC).

En el siguiente capítulo, se estudiará este proceso en una ley laboral de un Estado nacional determinado, a fin de visibilizar como un trabajador nacional es a la vez sujeto de derecho regional y universal. Como análisis de un caso concreto en este sentido, se comentarán los puntos esenciales de la última reforma del régimen de trabajo rural argentino, con el fin de identificar los principales cambios producidos por la incorporación de estándares internacionales de derechos humanos, en general, y de DESC, en particular.

\section{NIVEL DE INCORPORACIÓN DE DESC EN LA REFORMA DEL RÉGIMEN DE TRABAJO AGRARIO ARGENTINA, LeY 26.727}

El objetivo de este apartado consiste en analizar el nivel incidencia de los DESC en este régimen nacional, como indicador de la reconfiguración del trabajador rural, como sujeto de derechos humanos, en el marco de un proceso de incorporación de estándares internacionales de DESC en las leyes de trabajo nacionales.

Las numerosas transformaciones de la agricultura argentina en las últimas décadas, en materia de tecnificación y reconversión de los modos de producción, fueron el marco propicio para la 
sanción del Nuevo Régimen Nacional de Trabajo Agrario, por la Ley $\mathrm{N}^{\circ}$ 26.727, que fue sancionado en 2011 y reglamentado en 2013.

El antiguo régimen determinaba que la normativa aplicable al contrato de trabajo agrario era la ley 22.248/80, sus normas reglamentarias, la voluntad de las partes y los usos y costumbres. Al mismo tiempo, mediante la reforma del art. 2, inc. 3, de la Ley de Contrato de Trabajo (en adelante LCT) 20.744/76, se excluyó a los trabajadores agrarios del régimen general de la mencionada ley.

Cierta doctrina, entiende que esa modificación significó un notorio retroceso para los trabajadores agrarios, ya que no sólo derogó las normas de índole legal sino también otras numerosas disposiciones que, a través del tripartismo (representación sindical, empleadora y estatal) que implicaba la Comisión Nacional de Trabajo Rural (cfr. Ley 13.020), se había ido elaborando a través del tiempo. (Senyk \& Ruiz, 2012, pág. 202).

En este contexto, una de las modificaciones más relevantes del Nuevo Régimen de Trabajo Agrícola es la reincorporación a la Ley de Contrato de Trabajo, como fuente jurídica subsidiaria aplicable al trabajo agrario (art. 2, inc. b). Por tanto, será de aplicación en todo lo que resulte compatible y no se oponga al régimen jurídico específico. Como así también, reconoce como fuente de regulación a los convenios y acuerdos colectivos (Leyes 14.250 y 23.546) y a las Resoluciones de la Comisión Nacional de Trabajo Agrario (CNTA) y de la Comisión Nacional de Trabajo Rural aún vigentes.

\section{IV.1. Régimen especial y/o aplicación de la LCT para el trabajo agrícola}

Esta modificación nos posiciona en el antiguo debate sobre la necesidad de una legislación específica para el trabajo agrario y luego, sobre la conveniencia de la aplicación, o no, y en qué medida, de la LCT al trabajo agrario. Entendemos que este debate se vincula con el art. 6 del PIDESC, ya la aplicación subsidiaria de 
la LCT implica las garantías a los derechos laborales del régimen laboral. En este sentido encontramos tres posturas: La partidaria de la aplicación de un régimen especial con la exclusión de la LCT, la que defiende la aplicación de la LCT y la regulación de los caracteres especiales mediante convenios colectivos y finalmente la que sostiene la necesidad de un régimen especial pero con la aplicación subsidiaria de la LCT.

En el primer caso, parte de la doctrina sostiene que:

«la necesidad de una legislación especial para el trabajo agrario resulta evidente si se tienen en cuenta los caracteres propios de la actividad rural, que tornan inaplicables muchas de las disposiciones generales referidas al trabajo comercial o industrial con arreglo a las normas dictadas especialmente para ellos, primeramente por la ley 11.729 y el Decr. 33.302/45 y en la actualidad por la ley de Contrato de trabajo 20.744, reformada por la 21.297» (Brebbia Malanos, 2007, pág. 562).

Por tanto, el argumento que fundamentaría un régimen normativo especial y la exclusión de la LCT, se justificaría por las características especiales del trabajo rural. No obstante, al especificar estas notas características la segunda doctrina encuentra ciertas contradicciones que merecen ser destacados.

Desde una segunda postura, Mántaras considera como diferencias sustanciales que el trabajo industrial requiere mano de obra concentrada en los talleres bajo la inmediata dirección de los capataces, mientras que en el agrario no sucede lo mismo (Mántaras, 2007, pág. 563). Sin embargo, advierte que existen otras actividades que comparten con el trabajo agrícola el fenómeno de la estacionalidad, pero a los que resulta aplicable la LCT. Como ejemplos podemos mencionar la hotelería, de gastronomía, otros vinculados al turismo y a la industrialización de bienes primarios que tienen ritmos de producción discontinuos. En todos estos casos, las particularidades del oficio se han regulado mediante convenios colectivos y normas específicas de los organismos competentes, sin que se hayan ocasionado conflictos jurisprudenciales por dudas sobre la vigencia del régimen particular sobre el general. Es decir, 
que la temporalidad del trabajo se debe a los ciclos de producción de bienes o de servicios, y que por razones económicas, la contratación de los trabajadores se realiza de manera temporal, sin embargo, se les aplica la LCT.

Finalmente, el criterio adoptado por la ley establece un régimen especial pero con la aplicación subsidiaria de la LCT. Esta postura es la que entendemos como más acertada, por considerar que en Argentina, la exclusión de subsidiariedad de la LCT sobre el trabajo agrario, trajo como consecuencia la privación de numerosos beneficios para los trabajadores agrícolas vinculados a la seguridad social, en materia de beneficios jubilatorios, salario mínimo, ley de riesgos de trabajo, solo por mencionar algunos. Entendemos que con esta modificación, al reincorporar como fuente normativa de aplicación subsidiaria a la LCT para el trabajo agrícola, el legislador ha vuelto a considerar que sus características especiales no son un obstáculo para la aplicación de las normas del trabajo en general, que garantizan los derechos esenciales de todo trabajador, que es común a todos los sectores.

A continuación, se analizarán los puntos estratégicos de la última reforma del régimen de trabajo rural argentino, procurando identificar los principales cambios producidos por esta legislación desde la perspectiva de los DESC.

\section{IV.2. Trabajadores incluidos y excluidos}

Si bien es cierto que el único cambio que realiza el nuevo régimen respecto de trabajadores incluidos ${ }^{2}$ es excluír a los trabajadores que empaquen frutos o productos de otros productores, es importante destacar que existen establecimientos productivos

${ }^{2}$ Ley 26.727, art. 7: «Trabajadores rurales incluidos son: a) Manipulación y almacenamiento de cereales, oleaginosos, legumbres, hortalizas, semillas u otros frutos o productos agrarios, excepto que se realicen en establecimientos industriales. b) Tareas prestadas en ferias y remates de hacienda y c) Empaque de frutos y productos agrarios propios». 
que además de sembrar y cosechar ciertos productos agrícolas, también poseen plantas de procesamiento industrial.

Esta disposición resulta relevante ya que, al trabajador de cosecha de aceitunas, por ejemplo, se le aplica el régimen de trabajo agrícola, pero si ese mismo trabajador en el mismo establecimiento, es empleado en el empaque o procesamiento industrial de ese fruto, deberá aplicársele la normativa de la LCT. En este caso, entendemos que esta diversidad normativa, si bien implica un mejoramiento de derechos vinculado al art. 6, inc. 2, del PIDESC, en la práctica, implica un desafío importante en materia de control de correcta registración laboral ${ }^{3}$.

De este modo, los trabajadores de la industria vitivinícola se encuentran excluidos de la aplicación de la norma, ya que los convenios 154/91 y 85/89 comprenden tanto la actividad de los obreros de la viña como la de los operarios de bodega.

En este sentido, resulta conveniente comentar algunas consecuencias prácticas de esta exclusión. En el caso de los trabajadores temporarios, es habitual que luego de trabajar algunos meses en las cosechas de aceitunas o yerba, regido por las normas de trabajo agrícola, se trasladen a otra provincia para continuar su trabajo en las cosechas de uva (se rige por un convenio colectivo especial) limones o manzanas, que se rigen por otro convenio colectivo especial pero supletoriamente se les aplica el régimen general de la LCT. De esta manera, un mismo trabajador de cosecha que trabaje en este ciclo, será regido por tres legislaciones

${ }^{3}$ Ley 26.727, art. 3: «Trabajadores rurales excluidos: a) Personal afectado principalmente a actividades industriales, comerciales, turísticas, de transporte $\mathrm{o}$ servicios, aunque se desarrollaren en empresas o establecimientos mixtos, agrario-industriales o agrario-comerciales o de cualquier otra índole. b) Trabajador contratado para tareas ajenas a la actividad agraria. c) Trabajador del servicio doméstico. d) Personal administrativo. e) Personal dependiente del estado nacional, provincial o municipal. f) Al trabajador ocupado en tareas de cosecha y/o empaque de frutas, el que se regirá por la ley LCT $20.744 \mathrm{~g}$ ) Trabajadores comprendidos en convenciones colectivas de trabajo con relación a las actividades agrarias incluidas en el régimen de negociación».

Ius Humani, v. 4 (2014/15), p. 176 
diferentes a pesar de tratarse prácticamente de la misma actividad rural.

Estas exclusiones normativas adquieren una particular relevancia al momento de pensar en la correcta registración laboral de los trabajadores. Esto dificulta no solo el conocimiento y defensa de sus derechos a los trabajadores y su participación sindical $\left(\mathrm{UATRE}^{4}\right.$ en el primer caso y $\mathrm{SOEVA}^{5}$ en el otro), sino que también resulta complejo para elaborar un sistema de registración laboral eficaz de trabajo temporario rural. No solo en materia de control, sino también de registro y seguimiento estadístico al momento de pensar en políticas públicas eficaces para el sector. En este caso entendemos que la reforma del régimen de trabajo agrícola no ha mejorado sustancialmente los perjuicios en la exigibilidad de los derechos y de representación sindical que trae aparejada esta fragmentación normativa y sindical, que se vincula con los arts. 6 , inc. 2 y art. 8 , inc. 1 del PIDESC 6 .

En este contexto, también el debate teórico sobre la especialidad normativa para el trabajo agrario se ve relativizado ya que, indudablemente un trabajador de cosecha de uva es agrícola y su trabajo tiene esas características que justifican la creación de un régimen especial pero, no obstante, se le aplica la LCT y convenios colectivos específicos.

Estos trabajadores, también quedan fuera de los organismos de gestión control competentes para el trabajo agrario como el Registro Nacional de Trabajadores y Empleadores Agrícolas

\footnotetext{
${ }^{4}$ UATRE: Unión Argentina de Trabajadores Rurales y Estibadores.

5 SOEVA: Sindicato de Obreros y Empleados Vitivinícolas y Afines.

${ }^{6}$ PIDESC, art 6, inc. 2: «Entre las medidas que habrá de adoptar cada uno de los Estados Partes en el presente Pacto para lograr la plena efectividad de este derecho deberá figurar la orientación y formación técnico-profesional, la preparación de programas, normas y técnicas encaminadas a conseguir un desarrollo económico, social y cultural constante y la ocupación plena y productiva, en condiciones que garanticen las libertades políticas y económicas fundamentales de la persona humana».
} 
(RENATEA) y de las disposiciones de la Comisión Nacional de Trabajo Agrícola.

De lo anteriormente expuesto, se observa que existen ciertos puntos de quiebre en la legislación de trabajo agrícola difíciles de controlar, el de trabajo agrícola e industrial en un mismo establecimiento, y el de trabajo agrícola y LCT para trabajadores temporarios de cosecha de frutos en el primer caso y de frutas en el segundo. Este escenario de complejidad normativa tiene un impacto sobre la protección de los derechos de los trabajadores en materia de prestaciones de seguridad social vinculados a la correcta registración laboral que se ponen en tensión con la efectiva garantía de DESC.

\section{IV.3. Contrato de trabajo agrario}

En este sentido ${ }^{7}$, las innovaciones relevantes se vinculan con las modalidades de contratación de los trabajadores, con normas vinculadas al control de las cooperativas, a la prohibición de empresas de servicios de colocación de trabajadores y a la creación de bolsas de trabajo que comentaremos a continuación.

En su art. 14, la ley dispone que el Servicio Nacional de Inspección de Trabajo estará habilitado para ejercer el contralor de las cooperativas de trabajo, a los efectos de verificar el cumplimiento de las normas laborales y de la seguridad social, en relación con los trabajadores dependientes a su servicio, así como a los socios de ella que se desempeñaren en fraude a la ley laboral.

Con esta nueva facultad de control a cargo del Ministerio de Trabajo Nacional, la norma pretende reprimir situaciones habituales en las que se desnaturalizaba la figura jurídica de cooperativa que funcionaban con la dinámica de una empresa y

${ }^{7}$ Ley 26.727, art. 11: «Contrato de trabajo agrario es aquel por el cual una persona física se obliga a realizar actos, ejecutar obras o prestar servicios en el ámbito rural, mediante el pago de una remuneración en favor de otra y bajo su dependencia, persiguiera ésta o no fines de lucro, para la realización de tareas propias de la actividad agraria en cualquiera de sus especializaciones».

Ius Humani, v. 4 (2014/15), p. 178 
encubrían como socios a quienes en realidad eran trabajadores agrícolas.

Esta mecánica tenía el propósito de sustraerse, total o parcialmente, a la aplicación de la legislación del trabajo violentando el art. 101 y concordantes de la Ley de Cooperativas 20.337, y sus modificatorias. Pero ante todo, esta maniobra pretendía vulnerar los derechos laborales de los trabajadores, especialmente en materia de seguridad social. En consecuencia, la nueva ley prohibió a las cooperativas de trabajo actuar como empresas de provisión de trabajadores.

Otra de las problemáticas que el nuevo régimen pretende corregir con sus modificaciones, se relaciona con la tercerización de la contratación de mano de obra en el trabajo agrícola, ya sea por los llamados contratistas o cuadrilleros, como por las empresas de servicios de previsión de trabajadores. La estrategia empresaria de la tercerización implica simplificar la gestión, disminuir los costos fijos y evitar los riegos jurídicos de la contratación directa.

En este sentido, Víctor Rau afirma que:

«Los agentes contratistas de mano de obra aparecen desempeñando una de las funciones tradicionales de los intermediarios en los mercados de trabajo cual es la de organizar migraciones laborales temporarias entre los ámbitos de residencia de la mano de obra disponible y los lugares de trabajo distantes donde esta capacidad laboral será empleada» (2012, pág. 94). «Lo que a diferencia del empleo generado por contratación directa de las agroindustrias, el empleo ofrecido por los contratistas en Misiones se caracteriza por la generalizada informalidad y por un grado de precariedad laboral particularmente elevado» (2012, pág. 90).

Con la finalidad de prevenir este fenómeno, el art. 15 de la nueva ley prohíbe la actuación de las empresas de servicios para la provisión de trabajadores temporarios, agencias de colocación o cualquier otra empresa que provea trabajadores para la realización de las tareas y actividades incluidas en la ley y de aquellas que de 
cualquier otro modo brinden servicios propios de las agencias de colocación.

No obstante, si bien el nuevo régimen prohíbe las empresas de contratación, permite la actuación de contratistas, practica históricamente arraigada a la actividad. No obstante, el art. 15 debe leerse en concordancia con el art. 12 donde se establece que quienes contraten o subcontraten con terceros la realización de trabajos o servicios propios de actividades agrarias, serán solidariamente responsables del cumplimiento de las normas relativas al trabajo y de las obligaciones derivadas de los sistemas de la seguridad social, y de las obligaciones emergentes de la relación laboral y de su extinción.

Sobre este punto, con el objeto de reformular los mecanismos de contratación de mano de obra, en su art. 66, la ley establece el uso obligatorio del Servicio Federal de Empleo para los empleadores, para la contratación de Trabajadores Temporarios de la Actividad Agraria.

En concordancia con esta disposición, en el art. 69 se crean las bolsas de trabajo a cargo de las asociaciones sindicales a fin de que provean a los empleadores del personal necesario para la realización de las tareas temporarias en las actividades contempladas en la presente ley, conforme las resoluciones que a tal efecto dicte la Comisión Nacional de Trabajo Agrario (CNTA).

Del conjunto de estos artículos puede inferirse que la intención del legislador consiste en morigerar los efectos de falta o irregularidad registral, ocasionada por la tercerización de mano obra temporaria. Con este fin y mediante la creación del servicio federal de empleo pretende ejercer un mayor control en las condiciones de contratación de los trabajadores a fin de garantizar sus derechos laborales. Y si bien prohíbe las actividades de las empresas de contratación, a fin de evitar la tercerización, no lo hace con el contratismo explícitamente.

Por otro lado, al hacer obligatoria la contratación mediante el Servicio Federal de Empleo debilitaría esa modalidad de 
contratación tercerizada. Resulta significativo que si bien la Ley decide negar la administración del registro de trabajadores y empleadores al poder sindical (UATRE) de trabajadores agrícolas, determina que la administración de las bolsas de trabajo quede a cargo de las asociaciones sindicales con representación gremial. No obstante, a pesar de que la ley fue reglamentada, a la fecha, aún no han sido creados ni el Servicio Federal de Empleo ni las Bolsas de Trabajo.

Sin embargo, entendemos que aunque parcial y formalmente esta modificación del nuevo régimen ha constituido un avance en materia de incorporación del estándar internacional de garantía del derecho a un trabajo libremente escogido y aceptado del art. 6, inc. 1 , del PIDESC $^{8}$ y en materia de prevención del delito de reducción a la servidumbre y de tráfico de personas con fines de explotación laboral.

\section{IV.4. Modalidades de contratación}

En materia de modalidades de contratación de trabajo agrícola, el nuevo régimen crea una nueva categoría. A las antiguamente contempladas, de contrato permanente y contrato temporario, añade la de contrato permanente discontinuo. A continuación mencionaremos las notas más relevantes de la reforma en este punto.

\section{IV.4.1. Contrato de trabajo permanente continuo}

El contrato permanente continuo estaba contemplado por la ley 22.248, en el art. 63, al igual que la LCT, establecía un período de prueba de 90 días, pudiendo extinguirse el vínculo dentro de ese lapso sin responsabilidad indemnizatoria. Tanto el periodo de

\footnotetext{
${ }^{8}$ PIDESC, art. 6, inc. 1: «Los Estados Partes en el presente Pacto reconocen el derecho a trabajar, que comprende el derecho de toda persona a tener la oportunidad de ganarse la vida mediante un trabajo libremente escogido o aceptado, y tomarán medidas adecuadas para garantizar este derecho».
} 
prueba como la falta de indemnización por despido sin causa, son notas características de flexibilidad laboral y tuvieron un impacto negativo sobre el derecho económico de seguridad del ingreso de los trabajadores.

El nuevo régimen modifica este precepto determinando que el contrato de trabajo agrario se presume celebrado con carácter permanente. Por tanto, elimina el período de prueba, con lo cual en caso de ser despedido dentro de los 90 días, el trabajador deberá ser indemnizado, de conformidad con las pautas fijadas en la LCT.

\section{IV.4.2. Contrato de trabajo temporario}

La nueva ley no ha realizado innovaciones conceptuales con respecto a la definición de trabajo temporario con respecto a la anterior legislación ${ }^{9}$. Este tipo de contrato es análogo al de plazo determinado, del régimen general de la LCT y de prestación de servicios. Esto implica que finalizado el plazo señalado en el contrato, la relación laboral se extingue, al trabajador no se le debe indemnización y el empleador no tiene obligación alguna de volver a contratarlo nuevamente. Considerando que el mercado ocupacional agrícola argentino presenta un alto grado de irregularidad registral, esta categoría de trabajadores es la más vulnerable, social y jurídicamente, por factores como inseguridad en el ingreso, bajos niveles de ingreso y falta de cobertura de seguridad social, lo que se traduce como falta de garantía de DESC.

\section{IV.4.3. Contrato permanente discontinuo}

9 Ley 26.727, art. 17: «Contrato temporario es el que se origina por las necesidades de la explotación de carácter cíclico estacional o por procesos temporales propios de la actividad agrícola, pecuaria, forestal o de las restantes actividades comprendidas dentro del ámbito de aplicación de la presente ley, así como también, las que se realizaren en ferias y remates de hacienda. Se encuentran también comprendidos en esta categoría los trabajadores contratados para la realización de tareas ocasionales, accidentales o supletorias».

Ius Humani, v. 4 (2014/15), p. 182 
Desde el punto de vista normativo e institucional, las principales dificultades se presentan al intentar encontrar un equilibrio entre los intereses de los empleadores, la seguridad en el ingreso y la protección social de los trabajadores. Esto se debe a que, los productores requieren asalariados temporales y cíclicos para las cosechas, pero estos trabajadores necesitan una fuente laboral y de ingreso durante todo el año y la certeza de que el próximo año serán nuevamente contratados. Con la finalidad de armonizar estos intereses contrapuestos, el nuevo régimen crea un nuevo modo de contratación: la de contrato permanente discontinuo.

Esta nueva modalidad, enunciada en el art 18, implica que en los casos en que un trabajador temporario sea contratado por un mismo empleador más de una vez, de manera consecutiva, para la realización de tareas cíclicas o estacionales. En estos casos, el trabajador deja de ser temporario y adquiere los mismos derechos que los trabajadores permanentes. Si bien es cierto que esta medida constituye un avance indiscutible en lo referido a la protección del derecho económico de seguridad en el ingreso, vinculado al art. 7 inc. a.ii del PIDESC ${ }^{10}$, su efectividad depende de que las empresas no eviten contratar a los mismos trabajadores dos temporadas seguidas, como estrategia para reducir costos laborales. También conviene considerar que en un ámbito con altos índices de falta de registración laboral la eficiencia de esta medida se relativiza.

\section{IV.4.4. Prohibición de trabajo infantil}

Otro elemento que introduce la nueva ley explícitamente, es la modificación sobre trabajo de los menores de edad (arts. 55-57 y 59). En concordancia con la ley n ${ }^{\circ} 26.390$ de prohibición de trabajo infantil (B.O. 25/6/08), el nuevo régimen prohíbe el trabajo de menores de 16 años (antes de 14 años). Los menores, que tengan entre 16 y 18 años, solo pueden celebrar contratos de trabajo,

${ }^{10}$ PIDESC, art. 7, inc. a ii: «Condiciones de existencia dignas para ellos y para sus familias conforme a las disposiciones del presente Pacto». 
siempre que presenten: certificados de aptitud física, de escolaridad y de autorización de sus padres, o tutores. En cuanto a la jornada de trabajo no podrá ser superior a 6 horas diarias y 32 semanales y se prohíbe la jornada nocturna. Esta incorporación es el ejemplo más claro de incorporación de estándares internacionales de derechos humanos a la legislación nacional, ya que en materia de trabajo infantil los tratados establecen el límite en los 16 años. Este avance se vincula también con el art. 10, inc. 3, del PIDESC ${ }^{11}$.

\section{DERECHOS VINCULADOS A LA SEGURIDAD SOCIAL}

\section{V.1. Preaviso}

La nueva ley incorpora una serie de derechos orientados a lograr una mayor protección en materia de derechos a la seguridad social establecidos en el art 9 del PIDESC ${ }^{12}$. Uno de ellos es el preaviso. Se aplica tanto para los trabajadores permanentes continuos como para los discontinuos, asimilándose a la garantía laboral prevista en la $\mathrm{LCT}^{13}$. El propósito de este precepto es posibilitar al trabajador buscar con tiempo otra fuente de trabajo y en el caso del empleador, buscar un trabajador que ocupe el puesto vacante. En el primer caso, se protege la estabilidad del ingreso, en

${ }^{11}$ PIDESC, art. 10, inc. 3: «Se deben adoptar medidas especiales de protección y asistencia en favor de todos los niños y adolescentes, sin discriminación alguna por razón de filiación o cualquier otra condición. Debe protegerse a los niños y adolescentes contra la explotación económica y social. Su empleo en trabajos nocivos para su moral y salud, o en los cuales peligre su vida o se corra el riesgo de perjudicar su desarrollo normal, será sancionado por la ley. Los Estados deben establecer también límites de edad por debajo de los cuales quede prohibido y sancionado por la ley el empleo a sueldo de mano de obra infantil».

${ }^{12}$ PIDESC, art. 9: «Los Estados Partes en el presente Pacto reconocen el derecho de toda persona a la seguridad social, incluso al seguro social».

${ }^{13}$ Ley 26.727 , art. 21 , párrafo $2^{\circ}$. El preaviso implica la obligación, tanto para el empleador como para el trabajador de notificar previamente, cuando una de las partes ha decidido extinguir el vínculo laboral.

Ius Humani, v. 4 (2014/15), p. 184 
el segundo, se evitan los daños del lucro cesante que implicaría para el empleador un puesto de trabajo vacante.

Los plazos fijados son para el trabajador, de 15 días y para el empleador de 1 mes. La parte que omita el preaviso o lo otorgue de modo insuficiente deberá abonar a la otra una indemnización sustitutiva igual a la remuneración que le correspondería al trabajador por esos plazos. Si bien esta norma constituye un avance en materia de protección de derechos económicos, sin embargo, no se aplica a los trabajadores temporarios, que constituye el grupo de trabajadores más vulnerables.

\section{V.2. Indemnización por antigüedad}

Con respecto a los rubros indemnizatorios en el caso del trabajador permanente continuo (arts. 16 y 22), se asimilan a lo dispuesto en el título XII del régimen general de la LCT 20.744, que trata sobre la extinción del contrato de trabajo. Esta asimilación ha beneficiado a los trabajadores e implica, en nuestra opinión, un ejemplo de cómo el régimen especial de trabajo agrícola puede respetarse sin dejar de garantizar los derechos laborales esenciales del general.

La misma normativa se aplica al personal permanente discontinuo lo que significa una notable mejora para los trabajadores de esta categoría, ya que en materia de extinción de contratos de trabajo sin justa causa, la ley 22.248 no lo había contemplado. Los trabajadores que hoy forman parte de esta categoría, antes de la ley, no recibían remuneración por este concepto, ya que se consideraban solo temporarios y su contrato de trabajo se extinguía al final de cada cosecha, aunque trabajaran años consecutivos para el mismo empleador (art. 21).

El nuevo régimen, también incorpora para esta categoría la acción de daños y perjuicios en los casos de despido sin justa causa. En este caso el trabajador tiene derecho, además de las indemnizaciones previstas en el Título XII de la ley 20.744 (1976) 
y sus modificatorias, a la de daños y perjuicios provenientes del derecho común.

Con respecto al personal temporario, en caso de extinción de contrato de trabajo sin justa causa, el nuevo régimen (art. 20) ha incorporado una mejora elevando de $5 \%$ al $10 \%$ del total de las remuneraciones devengadas, además del sueldo anual complementario (SAC) y el proporcional de vacaciones, dependiendo de los meses trabajados que se mantienen como antes.

\section{V.3. Remuneraciones}

En este sentido, la nueva ley en su art. 32 establece que las remuneraciones mínimas serán fijadas por Comisión Nacional de Trabajo Agrario, y no podrán ser inferiores al salario mínimo vital y móvil vigente, del régimen general de la LCT. La novedad en este sentido, está dada por fijar como base el salario mínimo al fijado por el régimen general. Esta reforma pretende evitar que la especialidad del régimen agrario se convierta en un perjuicio para los trabajadores. Cabe destacar que si se habla de salario mínimo, este no se aplica a los trabajadores temporarios, a quienes se paga a destajo, por rendimiento, por la cantidad de unidades cosechadas. El pago se realiza semanal o quincenalmente. Esta precarización, producto de la falta de salario mínimo en este sector, lo posiciona en una peor situación frente al resto de los trabajadores, debido a la inseguridad del ingreso, no sólo por la contingencia de su continuidad laboral en la próxima cosecha, sino también por la imposibilidad de tener un ingreso mínimo garantizado.

Esta situación, los coloca en una situación de falta de capacidad de negociación laboral sobre la que inciden otras variables que caracterizan a este sector, como la falta de registración laboral, de seguridad en la continuidad laboral y sobre sus condiciones de vida. Estos factores contribuyen a reproducir las causas que originan su situación de pobreza estructural, que agrava su vulnerabilidad social y jurídica.

Compartimos la idea de que: 
«El salario mínimo es además un instrumento de política pública para contribuir a la reducción de la pobreza y la desigualdad en la distribución del ingreso. A diferencia de otras medidas que se pueden implementar para el logro de esos objetivos, no implica un compromiso fiscal equivalente sobre el erario público ya que se trata de una norma destinada a regir primordialmente la actividad económica en el sector privado» (Groisman, 2012, pág.10).

\section{V.4. Beneficio jubilatorio}

Quienes estén incluidos en este nuevo régimen, tendrán derecho a la jubilación ordinaria con 57 años de edad, sin distinción de sexo, en tanto acrediten 25 años de servicios con aportes. En este sentido, en el nuevo régimen de trabajo agrícola la edad requerida para percibir los beneficios jubilatorios es menor que en el régimen general de la LCT, que en su art. 252, la fija en 60 para las mujeres y 65 para los varones. No obstante, el mercado de trabajo agrario está particularmente afectado por la falta de registración laboral, razón por la cual, la acreditación de 25 años de servicios con aportes, es difícil para los trabajadores permanentes continuos, y mucho más para los trabajadores temporarios y permanentes discontinuos.

$\mathrm{Al}$ intentar comprender las posibles causas de la persistencia de la pobreza en el trabajo temporario agrícola, podríamos relacionarla con la precariedad laboral. En este sentido, podemos mencionar aspectos relacionados con las características de la actividad como:

«La estacionalidad productiva y la discontinuidad laboral que genera, y algunas vinculadas a la regulación social de estos mercados de trabajo que se expresan en menores niveles de ingresos, pago a destajo, mayor informalidad en las contrataciones y en las condiciones sanitarias, transporte y vivienda» (Fabio \& Neiman, 2010, pág. 79). 


\section{REgISTRO NACIONAL DE TRABAJADORES Y EMPLEADORES AgraRios (RENATEA)}

Finalmente, comentaremos una de las reformas esenciales del nuevo régimen. En el art. 106 deja sin efecto al Registro Nacional de trabajadores Rurales y Empleadores (RENATRE) y crea el RENATEA que asumió las funciones del anterior. El RENATEA es un ente autárquico, bajo la jurisdicción del Ministerio de Trabajo, Empleo y Seguridad Social (MTEySS). Deben inscribirse en el mismo, todos los empleadores y trabajadores agrarios. Sus principales funciones son jerarquizar a los trabajadores agrarios, mejorar las relaciones laborales del sector agrario, expedir la libreta de trabajo agrario, otorgar la prestación económica por desempleo, otorgar las prestaciones medico asistenciales, pagar las asignaciones familiares y fiscalizar el cumplimiento de la normativa de trabajo agrario.

El cambio fundamental de esta disposición consistió sustraer de la competencia del sindicato (UATRE) al anterior RENATRE reemplazándolo por uno nuevo, el RENATEA, bajo la directa jurisdicción del Estado nacional, a fin de conciliar el ámbito de registro y control con el de generación de políticas públicas.

\section{CONCLUSIONES}

1. En las últimas décadas la incidencia del proceso de globalización y de incipiente integración regional en América Latina, ha reconfigurado los modos de producción y la realidad agrícola en Argentina. Estos fenómenos de transnacionalización han influido también en el mercado de trabajo rural, generando una serie de transformaciones considerables en materia de derechos humanos.

2. Estos procesos de integración han tenido su correlato en el mundo jurídico, con un marcado desarrollo en materia de DESC en el mundo del trabajo, dentro del marco del Sistema Interamericano 
de Derechos Humanos. De este modo, la normativa supranacional relacionada con el progreso normativo e institucional de organismos de integración regional como MERCOSUR, permite hablar de la internacionalización de los derechos de los trabajadores a nivel regional y supranacional.

3. La crisis del constitucionalismo moderno, frente a los procesos de integración regional y de globalización, ha generado un debate teórico sobre la transformación del concepto de ciudadanía (demos). Este debate, es aplicable también a la condición de trabajador de cada ciudadano. El proceso de internacionalización también se manifiesta en la transformación del concepto de trabajador como sujeto de derecho nacional, en tanto que asimila su condición a la de sujeto de derecho regional y universal.

4. En consonancia con esta reconfiguración, la última reforma del régimen de trabajo agrario argentino, ha realizado importantes avances en materia de incorporación de estándares internacionales de derechos humanos a la legislación nacional en esta materia. Pueden mencionarse como ejemplos, la protección contra el trabajo infantil y ciertos progresos sobre estabilidad en el ingreso y derechos de seguridad social. No obstante, su principal debilidad, al momento de pensar en su aplicación, consiste en que el ejercicio de esos derechos se ha establecido desde el presupuesto del trabajo registrado, en un sector caracterizado por un alto nivel de irregularidad registral.

5. Finalmente, de lo anteriormente expuesto se infiere que esta reconfiguración del trabajador rural, como sujeto de derecho regional y universal, es principalmente normativa y las transformaciones institucionales y de hecho son aún incipientes. Sin embargo, la visibilidad de este debate teórico es relevante, ya que resulta esencial para dar continuidad a la incorporación de los estándares internacionales de derechos humanos en la normativa nacional, y para lograr una progresiva transformación institucional. 
Ius Humani, v. 4 (2014/15), p. 190 


\section{REFERENCIAS}

Ackerman, B. (1998). We the people. Transformation, vol. 2. Cambridge: Belknap Press of Harvard University Press.

Bendini, M., Radonich, M. \& Steimberger, N. (1999). De golondrinas y otros migrantes. Buenos Aires: La Colmena.

Brebbia, F. \& Malanos, N. (2007). Derecho agrario. Buenos Aires: Astrea.

Bustos Gisbert, R. (2005). La constitución red: Un estudio sobre supraestatalidad y constitución. Bilbao: Instituto Vasco de Educación Pública (IVAP).

Cascajo Castro, J.L. (2003). «Notas para un breve ensayo sobre constitución e interpretación constitucional». Estudios de Deusto, 51, 1. Tomado del sitio Web http://revista-estudios.deusto.es/index.php/estudios deusto/article/view/408/472 (consultado el 23-XI-2013).

Fabio F. \& Neiman M. (2010). «Precariedad en los mercados de trabajo rurales. Agricultura y Familias en Valle de Uco». En Busso, M. \& Perez, P. (eds.). La Corrosión del trabajo. Estudios sobre informalidad y precariedad laboral. Buenos Aires: Miño y Dávila.

Ferrajoli, L. (2003). «Pasado y futuro del estado de derecho». En Carbonell, M. (ed.), Neo constitucionalismo(s). Madrid: Trotta.

Garrido, C. y Peres, W. (1998). Grandes empresas y grupos industriales latinoamericanos. México, D.F: Siglo Veintiuno Editores.

Gonzáles Morales, F. (2013). Sistema interamericano de derechos humanos. Valencia: Tirant lo Blanch.

Groisman, F. (2012). «Salario mínimo y empleo en Argentina». Revista de economía política de Buenos Aires, año 6, vol. 11, pp. 9-47.

Haberle P. (2002). Pluralismo y Constitución. Estudios de teoría constitucional de la sociedad abierta. Madrid: Tecnos.

Habermas, J. (1997). «Replay to Grimm». En Gowan, P. \& Andersen, P. (ed.), The question of Europe. Londres: Verso.

Mántaras, L. M. (2007). El trabajo en las explotaciones rurales y sus diferencias con el trabajo industrial. Santa Fe: Universidad Nacional del Litoral.

Neiman, G. (2001). Trabajo de campo. Producción, tecnología y empleo en el medio rural. Buenos Aires: CICCUS. 
Neiman, G. \& Quaranta, G. (2000). «Restructuración de la producción y flexibilidad funcional del trabajo agrícola en la Argentina». Tomado de http://relet.iesp.uerj.br/Relet_12/Relet_12.pdf (consultado el 28XI-2013).

Rau, V. (2012). Cosechando yerba mate. Estructuras sociales de un mercado laboral agrario en el nordeste argentino. Buenos Aires: CICCUS.

Senyk, A. y Ruiz, Á. (2013). «La inspección laboral en el ámbito rural». Revista Derecho del Trabajo, I, 2. Tomado del sitio Web Infojus: DACF120145http://www.infojus.gov.ar/doctrina/dacf120145-ruizinspeccion_laboral_en_ambito.htm?1\&bsrc=ci (consultado el 6-VI2014).

Walker, N. (2002). «The idea of constitutional pluralism». Modern Law Review, 65 , pp. 317-359.

Ward, I. (2001). «Beyond Constitutionalism: the search of european political imagination». European Law Journal, 7, 1, págs.24-40.

Weiler, J.H.H. (1997). «Does Europe need a Constitution? Reflections on demos, tales and ethosin the german Maastricht decision». En Gowan, P. \& Andersen, P. (eds.), The question of Europe. Londres: Verso. 